\title{
Effect of Hand Grip Actions on Object Recognition Process: A Machine Learning based approach for Improved Motor Rehabilitation
}

\author{
*Anju Mishra \\ Shanu Sharma \\ Sanjay Kumar \\ Priya Ranjan \\ Amit Ujlayan
}

Accepted: 15 June 2020

\begin{abstract}
Brain computer interface (BCI) is the current trend in technology expansion as it provides an easy interface between human brain and machine. The demand for BCI based applications is growing tremendously and efforts are in progress to deploy BCI devices for real world applications. One of the widely known applications of BCI technology is rehabilitation in which BCI devices can provide various types of assistance to specially-abled persons. In this paper the effect of hand actions on objects is analyzed for motor related mental task. The proposed approach analysis electroencephalogram (EEG) based brain activity which was captured for images shown with different gripping actions on objects. The EEG recordings are first pre-processed, followed by extraction of epochs and frequency bands using discrete wavelet transform (DWT), afterwards feature extraction followed by training and classification steps are performed for classifying the grip action into congruent (correct) and incongruent (incorrect) grip categories. The proposed work makes use of average power and relative wavelet energy as discriminating features which are then fed to train an artificial neural network for automatically classifying the incoming EEG patterns into correct or incorrect object hand grips. The performance evaluation of proposed system is done on real EEG data set obtained from 14 subjects. Experimental results have shown an accuracy of $75 \%$. Also, to evaluate the effectiveness of our work, a comparison of our work with other state of art works reported by different authors is presented at the end. The results show the effectiveness of proposed approach and suggest further that the system can be used for analyse and train subjects having motorrelated disabilities for perceiving correct or incorrect hand grips on objects.
\end{abstract}

Keywords: Brain computer interfaces; Machine Learning, EEG, Hand action, Neural Network

\footnotetext{
*Anju Mishra

Department of IT, Amity University Uttar Pradesh, Noida, 201313, Uttar

Pradesh, India E-mail: amishra1@ amity.edu

Shanu Sharma

Department of CSE, Amity University Uttar Pradesh, Noida, 201313 Uttar

Pradesh, E-mail: ssharma6@amity.edu

Sanjay Kumar

Oxford Brookes University, Oxford, UK E-

mail: skumar@ brookes.ac.uk

Priya Ranjan

Department of EEE, Amity University Uttar Pradesh, Noida, Uttar

Pradesh, India E-mail: pranjan@amity.edu

Amit Ujlayan

School of Vocational Studies, Gautam Budhha University, Greater Noida, Uttar Pradesh, India

E-mail: amitujlayan@gbu.ac.in
} 


\section{Introduction}

Brain Computer Interface (BCI) is an emerging field of study, which can be used as a communication medium between a human and the computer. There are numerous possibilities and application areas for BCI as they provide an easy interface to understand the functioning of human brain. BCI has applications in different fields such as medical, industrial, experimental psychology and neurorehabilitation to name a few [1]. The most effective way to understand the functioning of human brain is by analyzing electroencephalogram recordings, commonly known as EEG data. The EEG recordings contain cortical potentials, which occur during various mental processes [2]. These signals comprise of different frequency sub-bands: Delta $(4 \mathrm{~Hz})$, Theta $(4-7 \mathrm{~Hz})$, Alpha or mu (8-12 Hz), Beta (12-30), and Gamma (30-100 Hz) bands, to facilitate ease of analysis. Studies presented in [3,4] found out that mu and beta rhythms are more sensitive to correct and incorrect hand grips and respond strongly especially over motor and pre-motor cortex areas of brain. Particularly the event related desynchronization of mu and beta rhythms were found to be more profound for congruent grip on objects rather than for incongruent grip [4]. Here, we present a fully automated system capable of sensing the correctness of grip response over familiar objects by analyzing EEG data. The proposed system takes EEG data and applies preprocessing over it which includes re-referencing of EEG data over left and right mastoid electrodes using EEGLAB software in MATLAB environment. After this, epoch extraction was performed on the re-referenced data and 89 epochs were obtained. The epoched EEG signals were then filtered using Discrete Wavelet Transform (DWT) to isolate Alpha and Beta rhythms. Finally, features were extracted from Alpha and Beta rhythms and passed on to a neural network classifier for training. The proposed system can be used for training purpose in neuro rehabilitation of especially abled persons and can be used to develop a variety of BCI devices to control limb movements in robotic and prosthetic settings as well [5]. Main contribution of our work can be summarized as follows:

- There is a substantial difference between the perception of EEG patterns of different type of handgrip, i.e., correct and incorrect action on objects. Our work tries to establish the fact that these different perceptions can be captured in EEG patterns at different brain locations and certain band features can be used for developing a machine learning based approach for automatic classification of appropriate hand grip categories.

- We also show that Relative Wavelet Energy is strong enough as a feature to be fed into a neural network to capture the essential differences in congruence of handgrip for object affordance.

- Since, neural processing is a networked process where nearby neurons contribute equally in processing of different tasks, in proposed approach we made use of pooling nearby electrodes at three different brain regions to show which area(s) of brain activate more in terms of average energy content for object affordance tasks.

\section{Related Work}

In past years, EEG-based systems have shown significant contribution in the development of BCI-based devices and in facilitating neuro-rehabilitation processes [6,7]. Machine learning techniques have also proven their worth in various fields including medical domain. Some of the previous works which made use of machine learning approaches in the mentioned field are further discussed here. Use of neural networks for EEG based epilepsy analysis can be found in [8-13]. In [8], authors proposed an automated system for epilepsy detection using neural network approach. The system used statistical measure approximate entropy (ApEn) as input feature. The authors found that the value of the ApEn decreases suddenly during an epileptic seizure. Similarly, a K- nearest neighbor and multilayer perceptron neural network (MLPNN) based decision system was proposed by Umut et al. for epilepsy treatment [13]. Further, use of support vector machine (SVM) classifier for EEG-based automated analysis of epilepsy can be found in [9]. The method involved extraction of multi scale key points in EEG signals based on difference of Gaussian filtered signals followed by feature extraction using Local binary patterns (LBPs). Use of different classifiers for classifying preictal and ictal conditions from EEG signals is given in [10]. Investigation of autism and driver fatigue based on EEG signals and neural networks can also be seen in [14-17]. In [14] a new computer aided diagnosis (CAD) of autism based on EEG signals is proposed where authors have used discrete DWT, entropy (En), and artificial neural network (ANN) to classify person as autistic or healthy. A Bayesian neural network classifier based driver fatigue versus alert state system is proposed in [16], where source separation was done using entropy 
rate bound minimization- ICA (ERBM-ICA) and features were extraction by autoregressive(AR) modeling. In [17] online cognitive failures in driving was assessed from EEG signals. Visual attentiveness of driver was analyzed by classifying EEG signals into alert and non-alert states. A type-2 fuzzy set induced neural classifier was used to remove the uncertainty in classification of motor planning. In other literature, neural networks have also been used in classification of different mental tasks. In [18] a BCI-based system was presented in which five types of mental tasks, i.e., baseline, mental letter composing, geometric figure rotation, math and visual counting were classified. For this classification task wavelet transform (WT), Fast Fourier transform (FFT) and principal component analysis (PCA) based features were extracted and ANN and SVM based classification was done. Deep learning has also been proven to be useful for classifying EEG patterns for variety of tasks such as motor imagery, mental workload, emotion recognition, seizure detection, event-related potential detection to name a few [19,20]. In [12] and [13] authors have presented the analysis of grip-based actions on object recognition process by examining the modulations in $m u$ rhythm during participant decisions to objects and nonobjects with congruent and incongruent grips. To analyze the response of neural excitation, event related desynchronization (ERD) is used as an index. It has been observed that $m u$ frequency band has higher ERD activity over motor scalp regions while performing decisions related to objects with congruent and incongruent gripping. The work presented in this paper is extension of work done by Sanjay et al. in [3,4]. Here, we present a fully automated system capable of sensing the correctness of grip response over familiar objects by analyzing EEG data. Further, the proposed work presented in this paper is structured as: Section 3 presents the methodology of our work along with dataset description and computational procedure. Various results obtained at different steps are explained in Section 4. Major Key findings of proposed work are underlined in Section 5 and paper is concluded in Section 6 along future implications.

\section{Experimental Details}

EEG recordings contain cortical potentials which occur during various mental processes. EEG signals are generally divided into different frequency bands: Delta $(4 \mathrm{~Hz})$, Theta $(4-7 \mathrm{~Hz})$, Alpha or mu $(8-12 \mathrm{~Hz})$, Beta (1230 ), and Gamma (30-100 Hz) bands, to facilitate ease of analysis. The present work features an automated model for analyzing changes in EEG responses corresponding to different objects gripped in hand with congruent and incongruent grips. The dataset and methodology used for the experimentation are discussed below:

\subsection{Dataset Description}

The proposed method is tested on real dataset presented in [3] and [4], which contains EEG recordings from a group of 14 people, out of which 3 were male and 11 were female. The participants were shown images of objects with congruent grip, objects with incongruent grip and no grip. Similarly, they were shown images of non-objects with congruent, incongruent and no grip. Sample images of stimuli for correct and incorrect grip on object are presented in Fig 1.

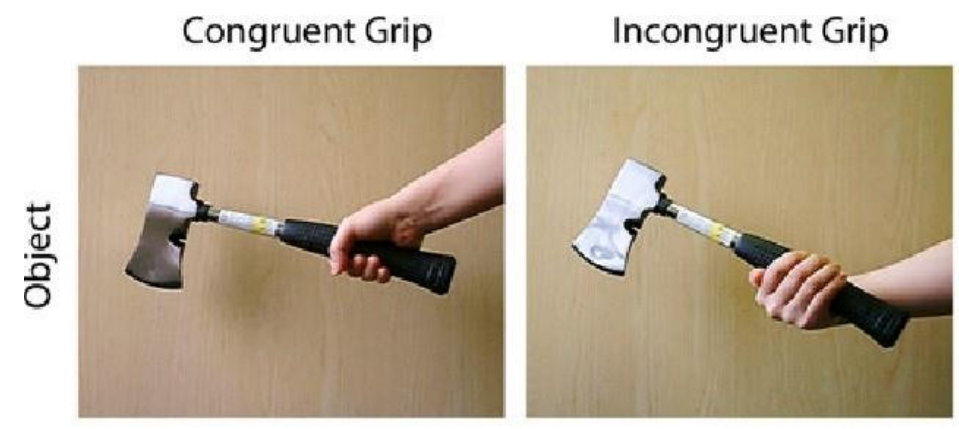

Fig. 1 Experimental Images of Congruent and Incongruent Hand Grips on Real Objects [3,4]

Participants have to respond quickly on stimuli to decide whether the object is real or not. The participants received a total of 180 stimuli of which 30 stimuli pertaining to objects and 30 were for non-object categories. Further, for each object and non-object category, three conditions were chosen: congruent grip, incongruent grip, and no handgrip. Before each task total 12 practice trials were shown to each participant, each trial started with 
fixation point for $1000 \mathrm{~ms}$ followed by the target stimulus for $1000 \mathrm{~ms}$. Participants were asked to make a response within $4000 \mathrm{~ms}$ after stimulus onset. For experimentation purpose, EEG data corresponding to congruent and incongruent grip on objects only is selected. The EEG data was recorded continuously with $\mathrm{Ag} / \mathrm{AgCl}$ electrodes placed on 128 scalp locations as shown in Fig. 2. The electrodes were placed according to 10-5 electrode system. Extra electrodes were used as references and ground. The signals were amplified and sampled at a rate of $1024 \mathrm{~Hz}$ using BioSemi Active-Two amplifiers.

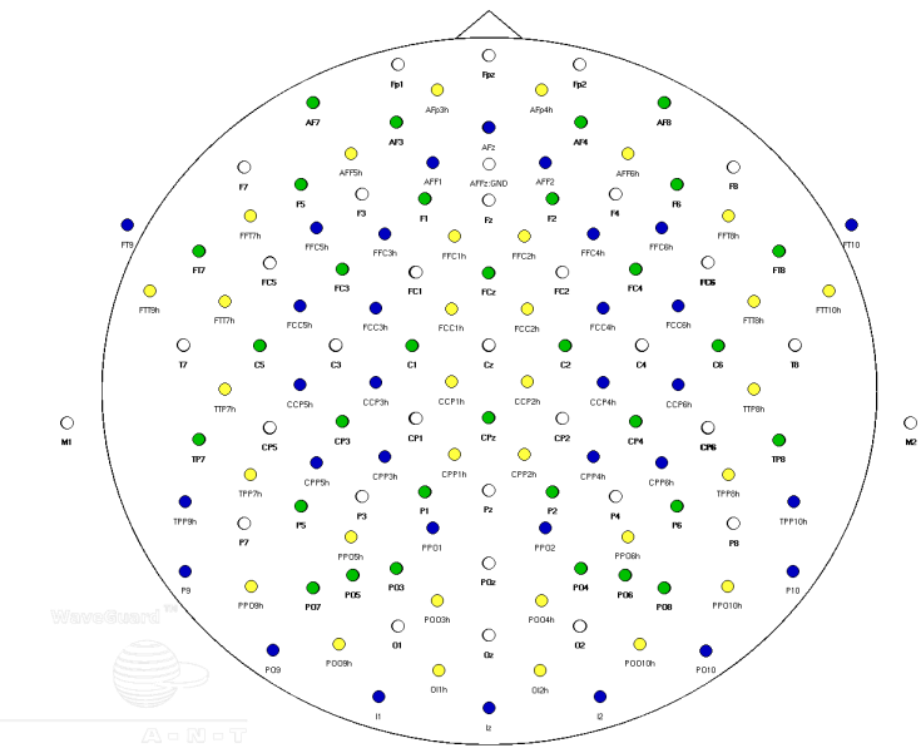

\subsection{Methodology}

Fig. 2 Standard 128 Electrode Positions on Scalp

The experimental procedure for analysis is presented in Fig. 3 and is explained below.

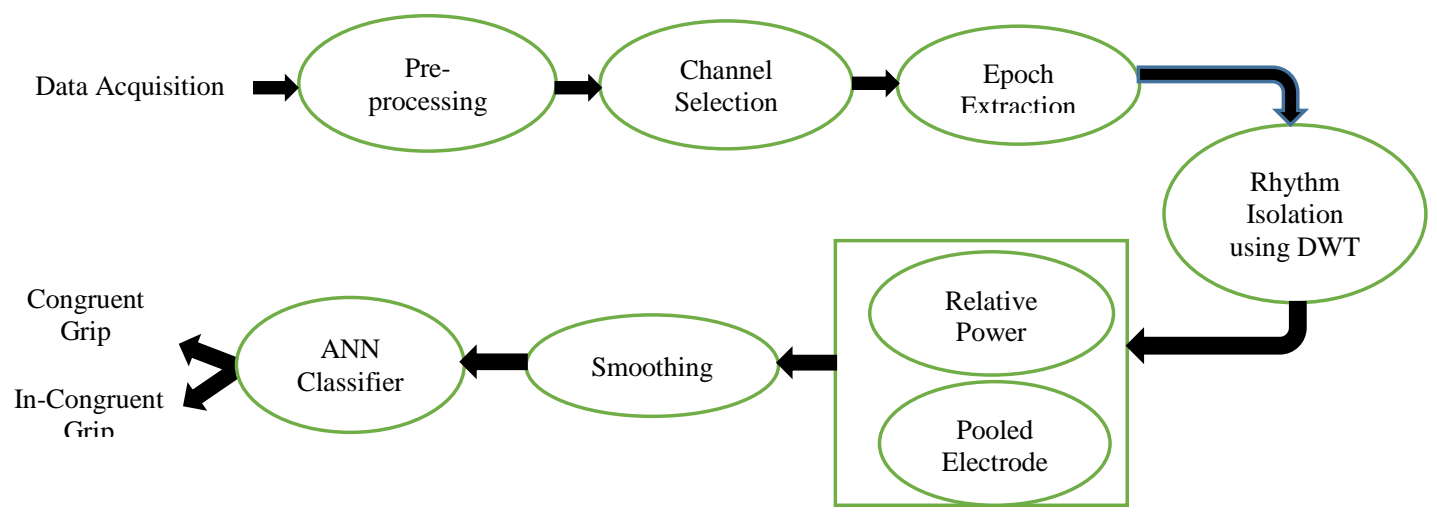

Fig. 3 Flow chart of Proposed Methodology

- Data Pre-Processing: The recorded EEG continuous data was first preprocessed using EEGLAB. This encompasses loading the data with reference to left and right mastoids respectively. The next step is to select first 128 channels from the EEG data as 129 to 134 channels are used for referencing and other purpose. After this step, the data is re-referenced at $\mathrm{Cz}$ electrode. This re-referencing step helps in subsidizing the noise content present in EEG signals. This noise reduction step is viable for correct identification of signal emitting area, i.e., the source of signal which in our case is the brain region [6]. The presence of noise is due to many factors such as heartbeats, eye flickers etc. occurring while recording the data with EEG electrodes [7]. 
The data contains 18 triggers associated with different events. First three triggers correspond to events associated with congruent, incongruent and no-grip responses over object category. While next three triggers are associated with congruent, incongruent and no grip responses over non-object category. The rest of the triggers are for reaction time responses. For this study, we have considered the first two triggers associated with object category only as our goal is to classify congruent and incongruent hand grip responses over objects for assessing object affordance from EEG signals. The data was then filtered between $3-40 \mathrm{~Hz}$ by using low pass and high pass filters available in EEGLAB. Next, notch filtering was done at $50 \mathrm{~Hz}$ to smooth out the sharp peaks in the signal.

- Channel Selection: Not all channels contribute equally in deciding that the grip response is correct or incorrect for a gripped object. Studies have shown that different brain areas respond to different types of activities. Therefore, while analyzing the EEG data we only considered EEG data recorded from occipital, motor and parietal areas of brain as most of the motor related activities are controlled from these areas of brain [6]. For each frequency sub-band there is a set of electrodes that responds well to achieve better classification results and these electrodes are associated with specific brain regions. It is also found from preliminary analysis that only certain frequency sub-bands of an EEG signal are important for analyzing certain task. For handgrip responses, it has been found that, out of the five sub-bands only the Alpha and Beta band respond well for recognizing handgrip actions on objects. This initial investigation suggests several hints to improve the classification task. For studying the effects of congruent and incongruent handgrip, we have analyzed the left and right brain hemispheres respectively and selected 24 electrodes from each of the left and right hemisphere. The 24 electrodes pertaining to each left and right hemisphere were taken from frontal brain region, central frontal region, central region, central parietal region and parietal region of brain areas.

Epochs with duration of $1000 \mathrm{~ms}$ before target onset and $1000 \mathrm{~ms}$ after target onset were extracted from continuous EEG signals. Activity for $1000 \mathrm{~ms}$ pre-stimulus was taken as the reference interval. Epochs were extracted with baseline from each left and right hemisphere. In our case a total of 28 epochs were generated. Epochs with values outside the range of $-100 \mu$ volt to $+100 \mu$-volt were discarded.

- Discrete Wavelet Transform based Rhythm Isolation: Band pass filtering of epochs in different frequency sub-bands is done for further analysis by using Discrete Wavelet Transform (DWT) [21]. Wavelet analysis was done to decompose the EEG signal into different frequency sub-bands. This transform is widely used for time frequency decomposition of acquired signals. Wavelet analysis falls into two categories: Continuous Wavelet Transform (CWT) and Discrete Wavelet Transform (DWT). CWT is useful for extracting event related potential (ERP) time-frequency features on nonstationary EEG signals and are suitable for effective feature se- lection which in turn results in significant classification accuracy. However, the drawback of using CWT is, it involves an excessive amount of calculations [22] [23. Therefore, from computational point of view DWT provides for faster calculation of constituting frequency bands.

In current research, for analyzing the EEG signal at different resolution, successive convolution of signals with high pass (HP) and low pass (LP) filters are done to decompose it into various high and low frequencies components respectively. Further, scaling of signal is performed using down sampling operation, which is a process of reducing the sampling rate of the signal. For performing wavelet transformation Daubechies-eight (db8) mother wavelet is used after testing different wavelets from Daubechies wavelet family. Further, as EEG data used in this experiment is having 1024 sampling rate thus, decomposition level was set at 8 to decompose the signal into required frequency bands. At each level of decomposition DWT outputs approximate $\left(\mathrm{A}_{\mathrm{i}}\right)$ and detailed coefficients $\left(\mathrm{D}_{\mathrm{i}}\right)$ using successive high pass and low pass filtering with down sampling rate of 2 [23] [24]. The approximation coefficients are then further decomposed as shown in Fig. 4 to extract localized information from the sub-band of detail coefficients.

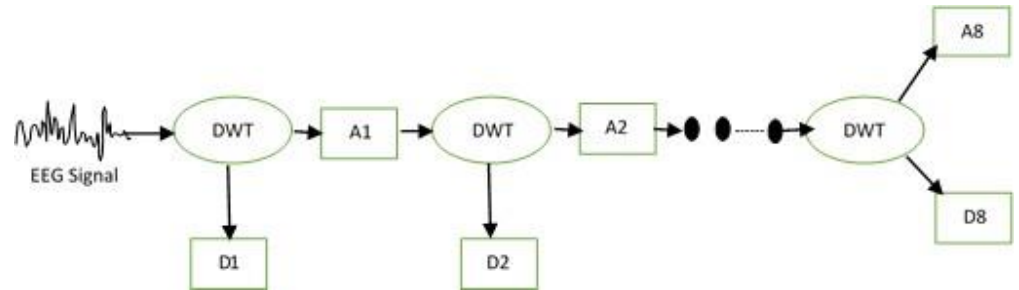

Fig. 4 EEG signal decomposition using 8-level DWT 
The motive behind extracting sub bands from an EEG band is because of the fact that specific rhythms show strong responses for specific type of events. It has been found that Alpha and Beta rhythms respond well for motor cortex activations. Therefore, Alpha and Beta bands were the focus of our study for hand grip actions. The frequency bands extracted from EEG signal using 8-level DWT are shown in Table 1. Each sub band, Delta (4 Hz), Theta (4-7 Hz), Alpha or mu (8-12 Hz), Beta (12-30), and Gamma (30-100 Hz) contributes to some specific characteristic of time series EEG signal.

Table 1 Frequency Sub-bands corresponding to Wavelet Coefficients

\begin{tabular}{lll}
\hline Frequency $(\mathrm{Hz})$ & EEG subband & Wavelet Coefficient \\
\hline $0-4$ & Delta & A8 \\
$4-7$ & Theta & D8 \\
$8-12$ & Alpha & D7 \\
$12-30$ & Beta & D6 \\
$30-100$ & Gamma & D5 \\
Above 100 & - & D1-D4 \\
\hline
\end{tabular}

- Feature Extraction: Various features used for training the neural network are explained below:

Relative Wavelet Energy: Previous studies suggest that a variety of features can be extracted from time series EEG signals viz. power, entropy and statistical features like mean, standard deviation, kurtosis etc. [25] [12]. Majority of previous studies have suggested the effectiveness of using entropy and power as major features for analyzing EEG signal data [10][13]. The fact that energy as a feature is strong enough for measuring complexity and regularity of time series data, makes it attractive to use for EEG data analysis. This motivated us to further investigate energy as a feature modality to analyze hand grip responses for object affordance. In our case, Relative Wavelet Energy (RWE) was chosen as a feature since it could help in selection of appropriate channel as not all channels have significant contribution in all type of activities. The relative wavelet energy was calculated using Equation (1).

$$
R W E=\frac{E_{\text {Alpha }}}{E_{\text {Total }}}
$$

Where $\mathrm{E}_{\mathrm{Alpha}}$ is the energy of Alpha band and $\mathrm{E}_{\text {Total }}$ is the total energy. Relative wavelet energy of Alpha band was computed for each of the 24 channels of left and right hemispheres respectively. A similar process was followed for beta band. Then the most promising channels (i.e. channels which were having high RWE difference) were selected. To help in the analysis process, we finally calculated the Baseline Normalized Features (F) using Equation 2, for normalizing the feature values w.r.t. the baseline signal [7].

$$
F=\frac{R W E_{\text {base }}-R W E_{\text {trial }}}{R W E_{\text {base }}}
$$

Next, to show the effect of nearby electrodes of specific brain regions for congruent hand grip and incongruent hand grip, nearby electrodes were pooled in six groups: three pools in each of the left and right hemisphere as shown in Figs. 5 and 6. Therefore 3 RWE features were obtained from three pooled electrode positions in left hemisphere of brain and 3 RWE features corresponding to the right hemisphere of brain. For our analysis, we have only considered the left brain hemisphere as all our participants were right handed. We have calculated RWE of Alpha and Beta Bands at 3 different positions of brain:

Pooled Position1: F1 F3 F5 FC1 FC3 FCC1H FCC3H FFC1H FFC3H FFC5h (10 Electrodes) Pooled Position 2: C1 C3 C5 CP1 CP3 CP5 CCP1H CCP3H CCP5H CPP1H CPP3H CPP5H (12 Electrodes)

Pooled Position 3: CP1 CP3 CP5 CCP1H CCP3H CCP5H CPP1H CPP3H CPP5 (9 Electrodes) 


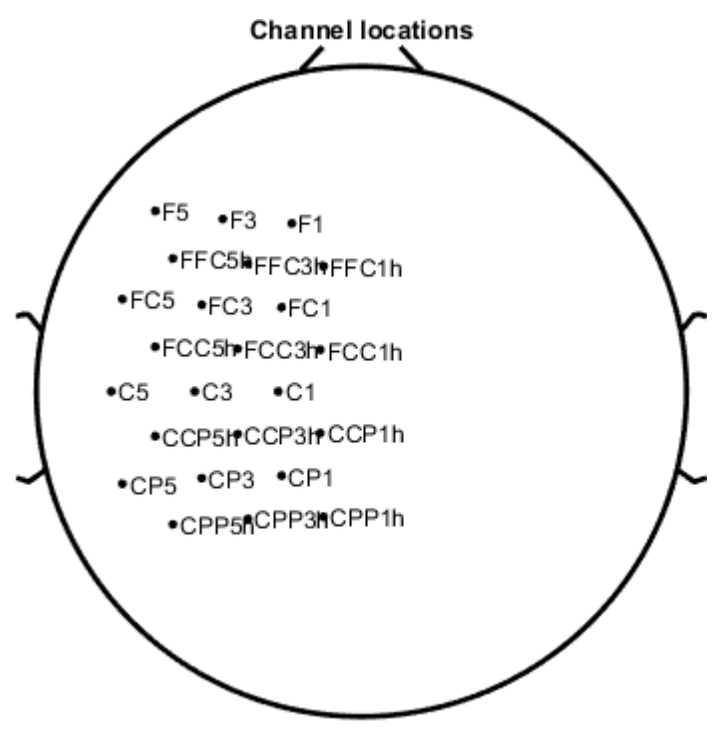

Fig. 5 Twenty Four electrodes in left hemisphere of brain

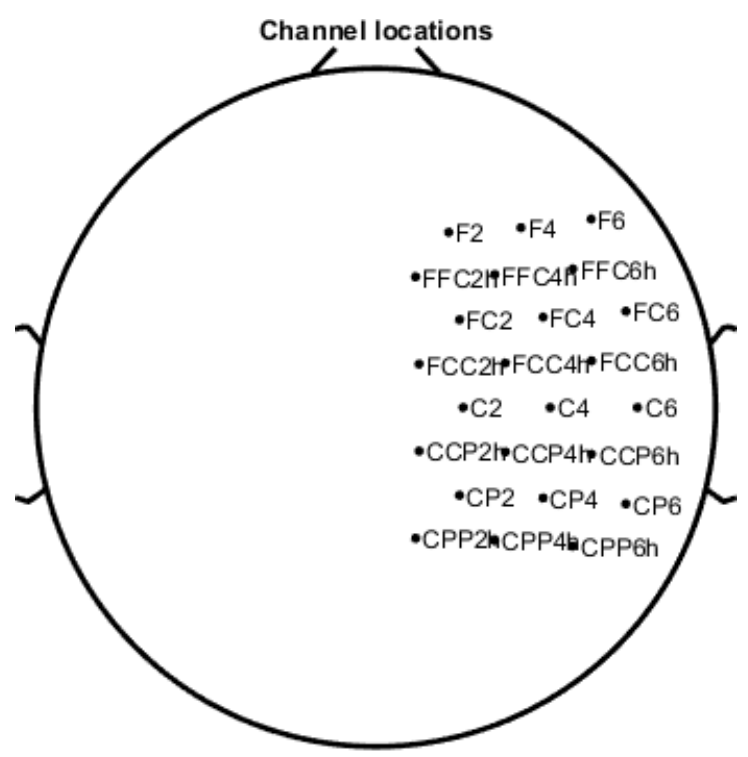

Fig. 6 Twenty Four electrodes in right hemisphere of brain

Power: Similarly, two more features were taken as the Alpha- and Beta- band's average power of selected twelve electrodes (refer to result section). Therefore, a total of five features were taken into account for classification purpose. The size of feature vector provided to the $\mathrm{NN}$ classifier was $5 \times 56$, where, five were the features and twenty eight were the epochs generated for each of the congruent and incongruent hand grip event, thereby making a $5 \times 56$ matrix, which was then fed to the neural network classifier. The next step after feature extraction was to classify EEG signal for congruent and incongruent grip on objects. For this, the feature vectors used to train an artificial neural network for classification purpose.

- Artificial Neural Network based Classification: Artificial neural networks (ANNs) are a widely adopted approach in the biomedical domain for classifying data. An ANN is an information processing system which imitates human cognitive processing. An ANN is a connected network of several neural computational units called artificial neurons. Each artificial neuron is a standalone information 
processing unit capable of processing received stimuli in parallel much like its biological counterparts. These neurons are organized into pre-defined layers of ANN [11]. The general architecture of artificial neural network is shown in Fig. 7. The network can be summarized mathematically using Equations 3 and 4:

or

$$
y=x_{1} \cdot w_{1}+x_{2} \cdot w_{2}+x_{3} \cdot w_{3} \ldots \ldots+x_{n} \cdot w_{n}
$$

$$
y=\sum_{i=1}^{n} x_{i} \cdot w_{i}+b
$$

where, $x_{i}$ are input to the network and $w_{i}$ are the corresponding weights. Here, $b$ is the bias factor which determines how likely the network will output a one. The net output $y$ is the sum of products of input matrix and weight matrix plus the bias. This net output is then fed to an activation function $\varphi($.$) to get$ the final output Y.

The final output is a function of $\mathrm{y}$ as given in Equation 5:

$$
Y=\varphi(\mathrm{y})
$$

Variety of activation functions are available in literature and the most widely used are the sigmoid function also called logistic sigmoid function and softmax functions. The logistic sigmoid function is very useful for binary classification and is used in hidden layers of proposed neural network. It can be expressed using Equation 6:

$$
f(x)=\operatorname{sigm}(x)=\frac{1}{1+\exp ^{(-x)}}
$$

The softmax function is generally used in last layer of an ANN, we also have used this function in the output layer of our ANN. The softmax function takes the form using Equation 7:

$$
f(x)_{i}=\frac{e^{z_{i}}}{\sum_{j=1}^{n} e^{z_{j}}}
$$

For $\mathrm{j}=1 \ldots \mathrm{n}$ and $z_{j}$ is the input vector. This function normalizes the input so that the output sums up to 1. The first layer is the input layer while last layer of ANN is the output layer. In between these two layers are a number of connected hidden layers that act like a series of transformation functions, which slowly maps input to the output of system. We have designed an ANN with four layers: one input layer, two hidden layers and an output layer. Each hidden layer contains five nodes and log sigmoid transfer function while output layer is designed to have two nodes with soft max transfer function as shown in Fig.7. The next section focuses on interpretation of results obtained during and after the experiment.

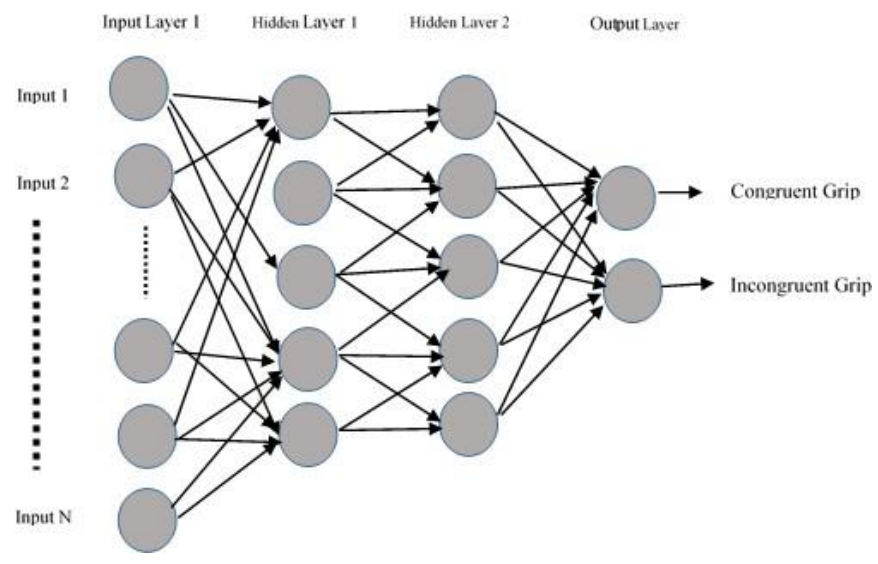

Fig. 7 Architecture of Artificial Neural Network 


\section{Results and Discussion}

Experimental results have shown that motor imagery-based perception of congruent and incongruent hand grips on different categories of objects produce substantial modulations in Alpha and Beta band rhythms which can be captured by a trained ANN and can further be classified into appropriate categories. Figs. 8 and 9 show the frequency spectrum of different bands obtained by DWT for congruent and incongruent hand grips respectively. By analyzing these spectra, we can easily conclude that there is a significant difference between the frequency values of Alpha and Beta band on congruence of hand grip.

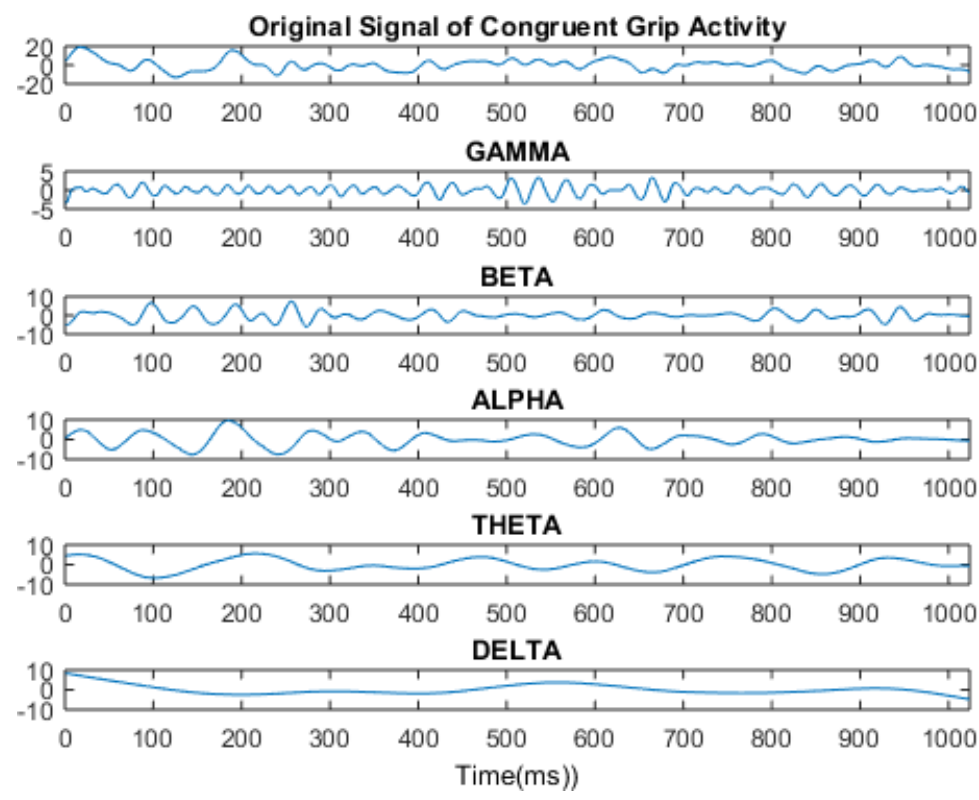

Fig. 8 Different frequency Sub-bands for Congruent Hand Grip
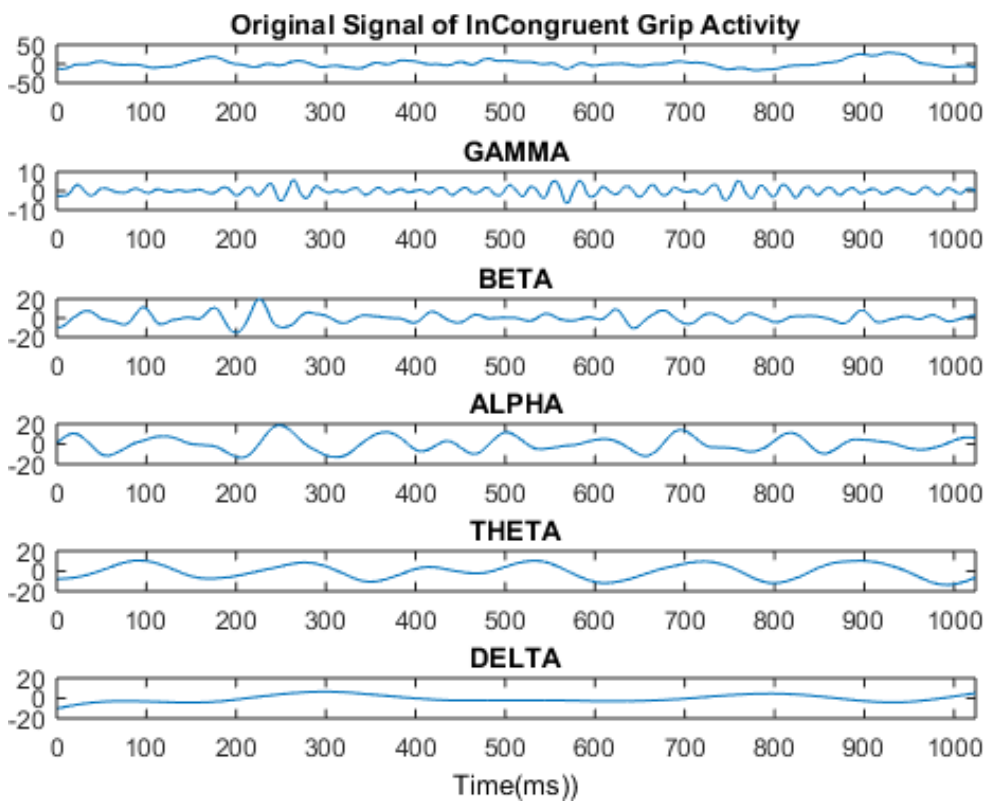

Fig. 9 Different frequency Sub-bands for Incongruent Hand Grip 
Features for ANN based classification were selected after Alpha and Beta power based analysis at pooled electrodes was done for each band respectively (Table 2). For power based analysis we have pooled the following twelve electrodes: CP1 CP3 CP5 CCP1H CCP3H CCP5H CPP1H CPP3H CPP5H C1 C3 and C5.

Table 2 Averaged Baseline Normalized Power (in \%) of Alpha and Beta bands for Congruent and Incongruent Grips

\begin{tabular}{|l|l|c|}
\hline $\begin{array}{l}\text { Average Baseline } \\
\text { Normalized Power }\end{array}$ & $\begin{array}{l}\text { Incongruent } \\
\text { Grip }\end{array}$ & Congruent Grip \\
\hline Alpha Power & 43.34 & 23.45 \\
\hline Beta Power & 14.83 & 32.31 \\
\hline
\end{tabular}

The graphs presented in Figs. 10 and 11 present the difference between average power of congruent and incongruent grips for Alpha and Beta bands for all epochs.

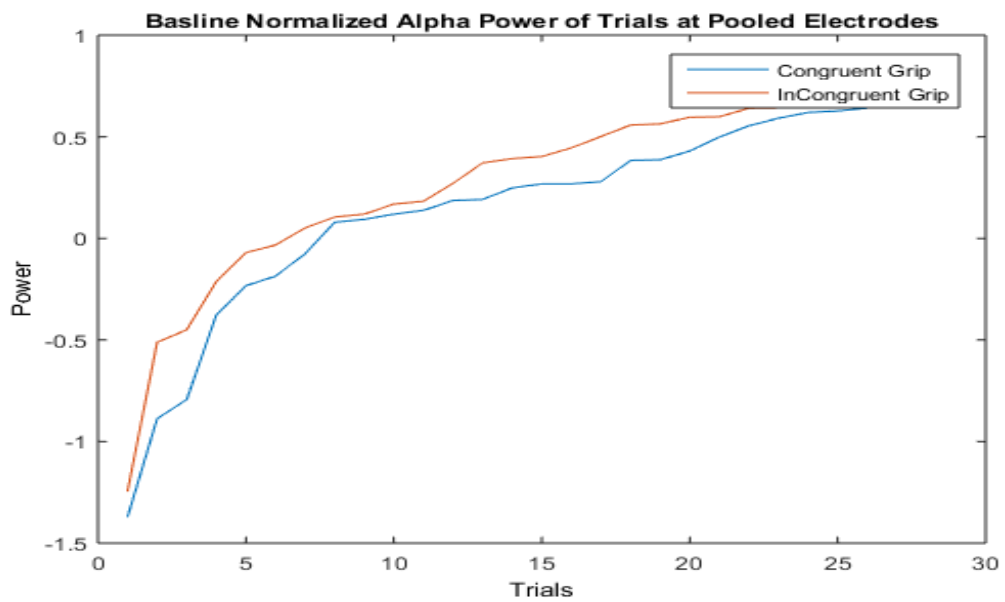

Fig. 10 Power Difference between Congruent and Incongruent Hand Grips for Alpha-band

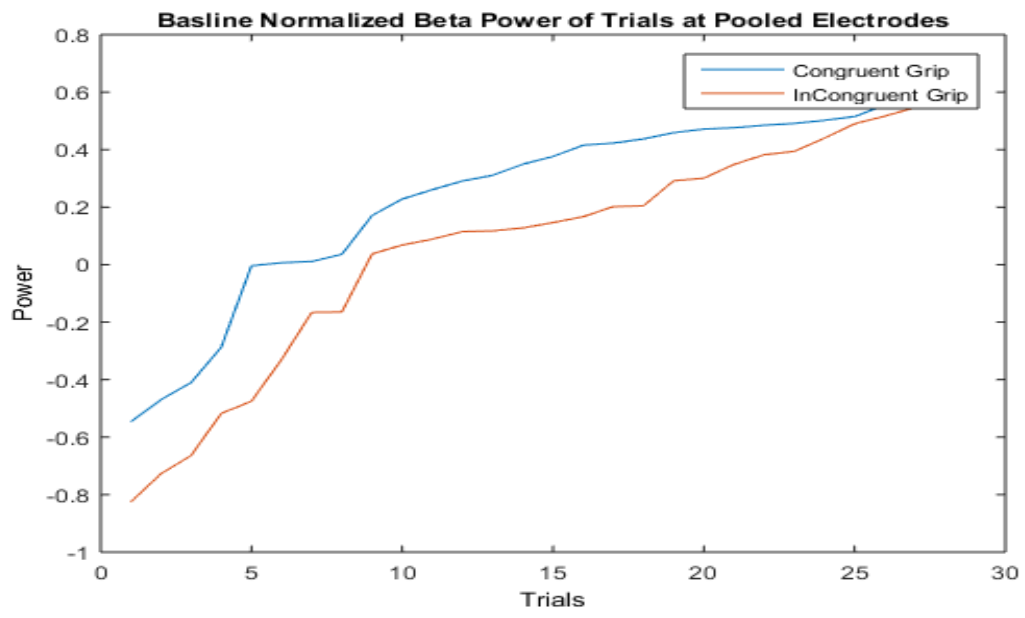

Fig. 11 Power Difference between Congruent and Incongruent Hand Grips for Beta-band

Three RWE features were obtained from three pooled electrode positions in left hemisphere of brain. For analysis purpose, we have only considered the left brain hemisphere. We have calculated RWE of Alpha and Beta Bands at three different positions of brain. Table 3 summarizes the difference between relative wavelet energy of Alpha and Beta bands for congruent and incongruent hand grips at three different brain locations in left hemisphere of brain. 
Table 3 Relative Wavelet Energy of Alpha and Beta bands for Congruent and Incongruent Grips at three locations

\begin{tabular}{|l|c|c|}
\hline \multirow{2}{*}{ Pooled Electrodes } & \multicolumn{2}{|c|}{ RWE at Alpha Band } \\
\cline { 2 - 3 } & Congruent Grip & $\begin{array}{l}\text { Incongruent } \\
\text { Grip }\end{array}$ \\
\hline $\begin{array}{l}\text { F1,F3,F5,FC1,FC3,FCC1H,FCC3H,FFC1H,FFC3H,FFC5h } \\
\text { P1,C3,C5,CP1,CP3,CP5,CCP1H,CCP3H,CCP5H,CPP1H,CP }\end{array}$ & 34.53 & 17.42 \\
\hline P3H,CPP5H & 20.81 & 33.68 \\
\hline $\begin{array}{l}\text { CP1,CP3,CP5,CCP1H,CCP3H,CCP5H,CPP1H,CPP3H,CPP5 } \\
\text { RWE at Beta Band }\end{array}$ & 31.11 & 8.4 \\
\hline
\end{tabular}

The graphs presented in Figs. 12, 13 and 14 show the effect of hand grips at three different pooled locations of left hemisphere of brain. First and second graph presents the difference in effect for two pooled locations corresponding to the Alpha-band. The third graph corresponds to the relative power difference for Beta-band.

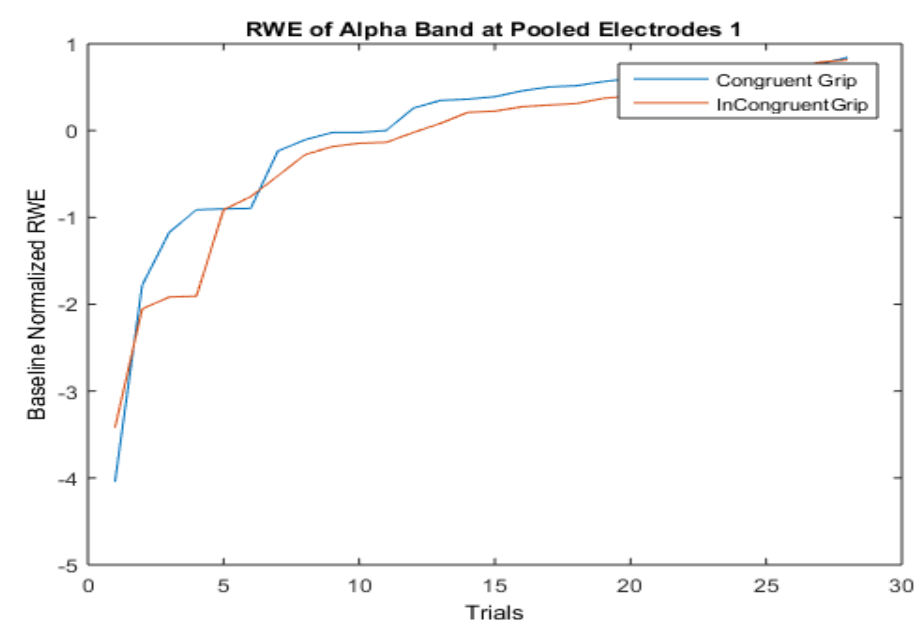

Fig. 12 Relative Wavelet Power Difference between Congruent and Incongruent Hand Grips for pooled location-1 (Alpha-band)

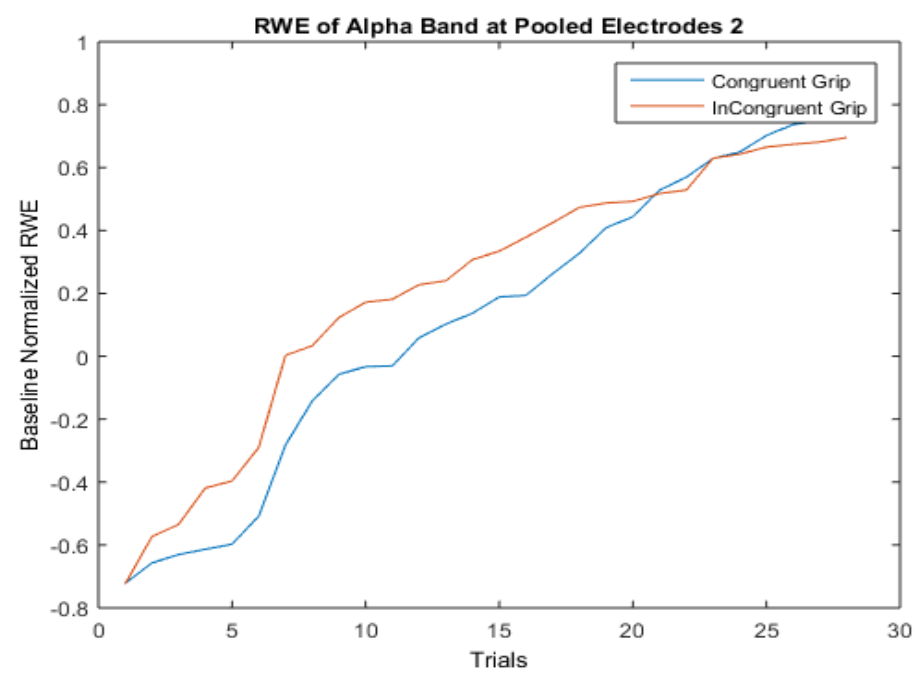

Fig. 13 Relative Wavelet Power Difference between Congruent and Incongruent Hand Grips for pooled location-2 (Alpha band) 


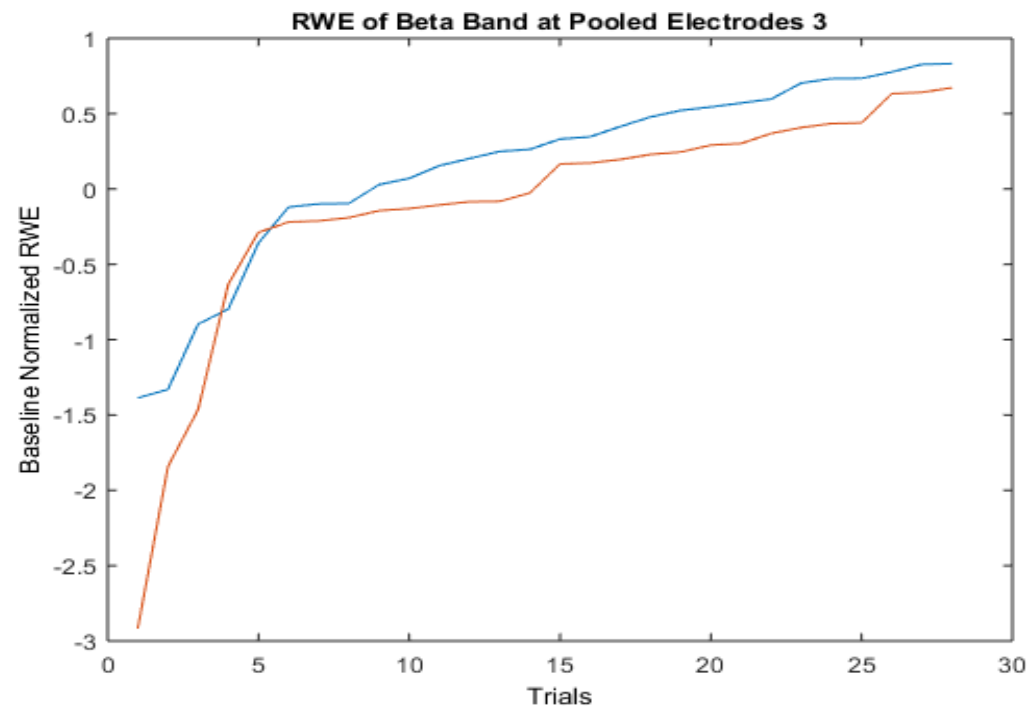

Fig. 14 Relative Wavelet Power Difference between Congruent and Incongruent Hand Grips for pooled location-3 (Betaband)

These graphs show the comparison of relative power of all epochs related to both events. These results clearly show that there is a significant deflection in the power of Alpha and Beta band waves with respect to the type of handgrip. This deflection is due to the differences in the power spectrum corresponding to EEG signal associated with each event. For analysis purpose, average power and relative power of Alpha and Beta bands were calculated for each event across all epochs and used as a feature of interest for classification purpose. These features are then fed to an ANN for classifying the event as congruent handgrip or incongruent handgrip. For the presented dataset, an accuracy of $75 \%$ was obtained by ANN for classifying EEG signals related to congruent and incongruent hand grips which is better than achieved by many authors.

Table 4 Comparison with different authors' work on Hand grasp task

\begin{tabular}{|lll|}
\hline Authors & Classifier Used & Accuracy \\
\hline Lange G. et. al. [26] & $\begin{array}{l}\text { linear discriminant } \\
\text { analysis (LDA) } \\
\text { linear discriminant } \\
\text { analysis (LDA) }\end{array}$ & $73.0 \%$ \\
Cho. J.H. et. al. [27] & $\begin{array}{l}\text { Probabilistic Neural } \\
\text { Network (PNN) }\end{array}$ & $61.96 \%$ \\
Ramadhan M.M. et. al. [28] & $\begin{array}{l}\text { Artificial Neural } \\
\text { Network (ANN) }\end{array}$ & $75.0 \%$ \\
Proposed Work & & \\
\hline
\end{tabular}

Table 4 presents a thorough comparison of performance of proposed approach with latest research works reported recently by different authors across the globe. Even though, these works made use of different types of hand grip data pertaining to imagined and executed hand grasp actions, these are inherently different in classification outcome, in our case, we simply training a classifier to identify whether the presented EEG recording represents a correctly gripped object or not. This comparison is to validate the effectiveness of our approach and suggests that achieving $75 \%$ accuracy is a good figure to start with, since, there are only minor differences in the energy contents of both types of signal.

\section{Key Findings}


The analysis presented in this paper successfully establishes the relationship between EEG patterns and object perception with different affordances. In this section, key findings of the proposed research are underlined:

- We presented that Alpha and Beta frequency waves have high deflection for two different gripping conditions over the motor cortex area of brain.

- We established that the neural processing is a networked process by analyzing the values of RWE and Average power of Alpha and Beta bands at three pooled electrode positions (see Tables 2 and 3). We found differential modulation of Alpha and Beta bands over the central-parietal motor area of the brain for appropriateness of grip processing.

- Then, using this analysis, we developed an ANN based automated model for classification of correct and incorrect hand grip on objects using EEG responses. Results presented also indicate that relative wavelet energy is a robust feature for developing correct/incorrect handgrip classification systems using EEG data.

\section{Conclusion}

In this paper, a novel analysis and automated method has been proposed to establish the relationship between EEG responses and object perception with different affordances. Though, a lot of work has been reported in literature for object affordance in the field of robotics, but only a little has been explored for developing systems that can help train persons with disabilities related to motor cortex area, specially having difficulties in perceiving the correctness of grip on objects. This work is a step forward in this direction and propose a classification system for determining correctness of grip from EEG signals. For analysis, EEG recordings of 14 subjects were taken while showing the images of objects with correct and incorrect grips. A series of operations, as presented in Section 3, were performed on EEG data including pre-processing, feature extraction, and classification. To find distinguishable features, average power and relative wavelet energy were analyzed thoroughly for each band and channel over different motor areas. We presented a rigorous analysis in section 4, which suggested that Alpha and Beta bands have shown substantial modulations over the central parietal motor area of the brain for different grips related to object perception. A set of specific features were extracted based on this analysis to propose an ANN based system for automatic classification of hand grip actions using EEG signals. Results suggests a robust neural correlate with object affordances during object perception and recognition process. The accuracy of proposed system was found to be $75 \%$, which will further motivate the researchers in field of neuro-rehabilitation to develop BCI based devices for assisting persons with impairment in using daily life objects ( e.g., in individuals with apraxia following stroke).

\section{Compliance with Ethical Standards}

Conflict of Interest: The authors declare that they have no conflict of interest.

Informed Consent: This article contains methodology that uses the data, which was collected previously in year 2011 by one of the mentioned authors Dr. Sanjay Kumar. Participants have provided written consent prior to participation. This previous study was also approved by the Local Ethics Committee of the University of Birmingham and conformed to the Declaration of Helsinki.

Animal Rights: This article does not contain any studies with animals performed by any of the authors.

\section{References}

1. Ramadan RA, Refat S, Elshahed MA, Ali RA (2015) Basics of Brain Computer Interface. DOI 10.1007/978-3-319-10978-7_2

2. Hassanien A and Azar A (2015) Brain Computer Interfaces: Current Trends and Applications, ISRL, vol. 74. Springer, Heidelberg. ISBN: 978-3-319-10977-0

3. Sanjay K, Eun YY, Glyn WH (2012) Perceptual and motor-based responses to hand actions on 
objects: evidence from ERPs. Experimental Brain Research 220:153-164

4. Sanjay K, Malcolm R, Glyn WH (2013) Mu rhythm desynchronization reveals motoric influences of hand action on object recognition. In: Front. Hum. Neurosci.

5. Alexis OR, Ioannisely BT, Hojjat A, John AB (2014) Combined corticospinal and reticulospinal effects on upper limb muscles. Neuroscience Letters 561:30-34, DOI https://doi.org/10.1016/j.neulet.2013.12.043

6. Chaudhari R and Galiyawala H. J (2017) A Review on Motor Imagery Signal Classification for BCI. Signal Processing: An International Journal (SPIJ), 11, 16-34.

7. Shedeed HA, Issa MF (2016) Brain-EEG Signal Classification Based on Data Normalization for Controlling a Robotic Arm. International Jour- nal of Tomography \& Simulation 29(1), URL http://www.ceser.in/ceserp/ index.php/ijts/article/view/3990

8. Srinivasan V, Eswaran C, \& Sriraam N (2007). Approximate Entropy-Based Epileptic EEG Detection Using Artificial Neural Networks. IEEE Transactions on Information Technology in Biomedicine, 11, 288-295.

9. Tiwari AK, Pachori RB, Kanhangad V, Panigrahi BK (2017) Automated Diagnosis of Epilepsy Using Key-Point-Based Local Binary Pattern of EEG Signals. IEEE Journal of Biomedical and Health Informatics 21(4):888-896, DOI 10.1109/JBHI. 2016.2589971

10. Acharya UR, Molinari F, Sree SV, Chattopadhyay S, Ng KH and Suri JS. (2012). Automated diagnosis of epileptic EEG using entropies. Biomed. Signal Proc. and Control, 7, 401-408.

11. AlSharabi K, Ibrahim S, Djemal R and Alsuwailem A. (2016). A DWT-entropy-ANN based architecture for epilepsy diagnosis using EEG signals. 2016 2nd International Conference on Advanced Technologies for Signal and Image Processing (ATSIP), 288-291.

12. Kannathal N, Lim CM, Acharya UR and Sadasivan PK. (2005). Entropies for detection of epilepsy in EEG. Computer methods and programs in biomedicine, 80 3, 187-94 .

13. Umut $\mathrm{O}$, Mahmut $\mathrm{H}$, Mahmut $\mathrm{O}$ (2011) EEG signals classification using the $\mathrm{K}$-means clustering and a multilayer perceptron neural network model. Expert Systems with Applications 38(10):13,47513,481, DOI https://doi. org/10.1016/j.eswa.2011.04.149

14. Ridha D, Khalil A, Sutrisno I, Abdullah A (2017) EEG-Based Computer Aided Diagnosis of Autism Spectrum Disorder Using Wavelet, Entropy, and ANN. In: BioMed research international

15. William JB, Adrienne LT, Helen TF, Charles AN (2011) EEG complexity as a biomarker for autism spectrum disorder risk. In: BMC medicine

16. Chai R, Naik GR, Nguyen TN, Ling SH, Tran Y, Craig A and Nguyen HT. (2017). Driver Fatigue Classification With Independent Component by Entropy Rate Bound Minimization Analysis in an EEG-Based System. IEEE Journal of Biomedical and Health Informatics, 21, 715-724.

17. Saha A, Konar A, and Nagar AK. (2017). EEG Analysis for Cognitive Failure Detection in Driving Using Type-2 Fuzzy Classifiers. IEEE Transactions on Emerging Topics in Computational Intelligence, 1, 437-453.

18. Bahy MME, Hosny M, Mohamed WA, Ibrahim S (2017) EEG Signal Classification Using Neural Network and Support Vector Machine in Brain Computer Interface. In: Proceedings of the International Conference on Advanced Intelligent Systems and Informatics 2016, Springer International Publishing, Cham, pp 246-256

19. Craik A, He Y and Contreras-Vidal J.L. (2019). Deep learning for electroencephalogram (EEG) classification tasks: a review. Journal of neural engineering, 16 3, 031001 .

20. Roy Y, Banville H, Albuquerque I, Gramfort A, Falk TH, Faubert J (2019). Deep learning-based electroencephalography analysis: a systematic review. Journal of neural engineering.

21. Subasi A (2007). EEG signal classification using wavelet feature extraction and a mixture of expert model. Expert Syst. Appl., 32, 1084-1093.

22. Akansu AN, Haddad RA (2001) Chapter 6 - Wavelet Transform. DOI https://doi.org/10.1016/B978012047141-6/50006-9

23. Kehtarnavaz N (2008) CHAPTER 7 - Frequency Domain Processing. DOI https://doi.org/10.1016/B978-0-12-374490-6.00007-6

24. Vivas EL, García-González A, Figueroa I, and Fuentes R.Q (2013) Discrete Wavelet transform and ANFIS classifier for Brain-Machine Interface based on EEG. In 2013 6th International Conference on Human System Interactions (HSI), (pp. 137-144). 
25. Shannon CE (2001) A Mathematical Theory of Communication. SIGMO- BILE Mob Comput Commun Rev 5(1):3-55, DOI 10.1145/584091.584093.

26. Gerrit Lange, Cheng Yee Low, Khairunnisa Johar, Fazah Akthar Hanapiah, Fadhlan Kamaruzaman, Classification of Electroencephalogram Data from Hand Grasp and Release Movements for BCI Controlled Prosthesis, Procedia Technology, Volume 26,2016, Pages 374-381, ISSN 2212-0173, https://doi.org/10.1016/j.protcy.2016.08.048.

27. Cho, Jeong-Hyun, Ji-Roon Jeong, Dong-Joo Kim, and Seong-Whan Lee. "A novel approach to classify natural grasp actions by estimating muscle activity patterns from EEG signals." In 2020 8th International Winter Conference on Brain-Computer Interface (BCI), pp. 1-4. IEEE, 2020.

28. M. M. Ramadhan, S. K. Wijaya and P. Prajitno, "Classification of EEG Signals from Motor Imagery of Hand Grasp Movement Based on Neural Network Approach," 2019 IEEE International Conference on Signals and Systems (ICSigSys), Bandung, Indonesia, 2019, pp. 92-96. 